\title{
IDEALS OF SQUARE SUMMABLE POWER SERIES. II
}

\section{JAMES ROVNYAK ${ }^{1}$}

The closed invariant subspaces of multiplication by $z$ in $H^{2}$ were determined by Beurling [1, Theorem IV, p. 253]. Vector generalizations of this theorem are known (Halmos [3] and the author [6]), but they involve an unnecessary use of analysis. We can now prove the theorem of [6] by purely algebraic and geometric methods. To emphasize these methods, we work with sequences, which we write as formal power series, rather than functions analytic in the unit disk.

Let $\mathcal{C}$ be a Hilbert space with elements denoted by $a, b, c, \cdots$, and with norm $|\cdot|$. If $b$ is a vector in $\mathfrak{e}$, then $b$ is the linear functional on $\mathfrak{C}$ such that $b a=\langle a, b\rangle$ for every $a$ in $\mathfrak{C}$. A formal power series is a sequence $\left(a_{0}, a_{1}, a_{2}, \cdots\right)$ written $f(z)=\sum a_{n} z^{n}$ with an indeterminate $z$. Let $f(z)=\sum a_{n} z^{n}$ and $g(z)=\sum b_{n} z^{n}$ be formal power series with coefficients $a_{n}$ and $b_{n}$ in $\mathcal{C}$; let $B(z)=\sum B_{n} z^{n}$ be a formal power series whose coefficients $B_{n}$ are (bounded) operators in $\mathfrak{e}$; let $\alpha$ be a complex number, and let $c$ be a vector in $\mathcal{C}$. Then $f(z)+g(z), \alpha f(z)$, $\bar{c} f(z)$, and $B(z) f(z)$ are the formal power series $\sum\left(a_{n}+b_{n}\right) z^{n}, \sum\left(\alpha a_{n}\right) z^{n}$, $\sum\left(\bar{c} a_{n}\right) z^{n}$, and $\sum\left(\sum_{k=0}^{n} B_{k} a_{n-k}\right) z^{n}$, respectively. A sequence $\left(f_{k}(z)\right)$ of formal power series with coefficients in $\mathcal{C}$ is said to be formally convergent if, for each $n=0,1,2, \cdots$, the corresponding sequence of $n$th coefficients is convergent. Let $\mathcal{C}(z)$ be the Hilbert space of formal power series $f(z)=\sum a_{n} z^{n}$ with coefficients $a_{n}$ in $\mathcal{C}$, such that

$$
\|f\|^{2}=\sum\left|a_{n}\right|^{2}<\infty \text {. }
$$

An ideal of $\mathfrak{C}(z)$ is a subspace $\mathfrak{T}$ of $\mathfrak{C}(z)$ which contains $z f(z)$ whenever it contains $f(z)$. The problem is to determine the closed ideals of $\mathfrak{C}(z)$. Let $B(z)$ be a formal power series whose coefficients are operators on $\mathfrak{C}$, and let $N(B)$ be the set of vectors $c$ in $\mathcal{e}$ such that $B(z) c=0$ identically. We write $\operatorname{Tr}(B)$ for the set of all products $B(z) f(z)$ with $f(z)$ in $\mathfrak{e}(z)$. The relevant condition on $B(z)$ is:

(1) $\left(z^{n} B(z) c_{n}\right)$ is an orthonormal set in $\mathcal{C}(z)$ whenever $\left(c_{n}\right)$ is a sequence of unit vectors in $\mathfrak{e}$ orthogonal to $N(B)$.

It is not difficult to show that (1) is equivalent to the assertion that $f(z) \rightarrow B(z) f(z)$ is a partial isometry of $\mathfrak{e}(z)$ into itself.

Received by the editors October 22, 1963.

${ }^{1}$ This work was partially supported by NSF Grant 3639 50-57. 
THEOREM. $A$ subset $\mathfrak{T l}$ of $\mathfrak{C}(z)$ is a closed ideal if and only if $\mathfrak{T l}=\mathfrak{T C}(B)$ for some formal power series $B(z)$ with operator coefficients which satisfies (1).

Proof. If a series $\sum f_{n}(z)$ converges in the metric of $\mathfrak{e}(z)$, then it converges formally, and the two limits coincide. For, the linear transformation from $\mathfrak{e}(z)$ to $\mathfrak{C}$, which takes a formal power series into its $n$th coefficient, is continuous.

Let $B(z)$ satisfy (1) and let $g(z)=B(z) f(z)$ be any element of $\operatorname{Tr}(B)$. If $f(z)=\sum a_{n} z^{n}$ is chosen so that its coefficients are orthogonal to $N(B)$, then $\sum z^{n} B(z) a_{n}$ converges in the metric of $\mathfrak{e}(z)$, and its norm squared is

$$
\sum\left\|z^{n} B(z) a_{n}\right\|^{2}=\sum\left|a_{n}\right|^{2}=\|f\|^{2} \text {. }
$$

But $\sum z^{n} B(z) a_{n}$ converges formally to $g(z)$, so by the above remark, $g(z)$ is in $\mathcal{C}(z)$ and $\|g\|=\|f\|$. We conclude easily that $\mathfrak{T}(B)$ is a closed ideal of $\mathfrak{C}(z)$.

Conversely, let TT be a closed ideal of $\mathfrak{C}(z)$ and let $B$ be the orthogonal complement in $\mathfrak{T}$ of the series $z f(z)$ with $f(z)$ in $\mathfrak{T}$. We begin by showing that the dimension of $B$ is no more than that of $\mathfrak{C}$. Since $B$ is a closed subspace of $\mathfrak{C}(z)$, the assertion is clear when $\mathcal{C}$ has infinite dimension. For in this case, $\mathfrak{C}$ and $\mathfrak{C}(z)$ have the same dimension. Now let $\mathcal{C}$ have finite dimension $r$ and let $c_{1}, \cdots, c_{r}$ be an orthonormal basis for $\mathcal{C}$. Suppose, to the contrary, that the dimension of $B$ is greater than the dimension of $\mathcal{C}$. Then there is an orthonormal set $f_{0}(z), \cdots, f_{r}(z)$ in $B$ containing $r+1$ elements. By the definition of $B$,

$$
\left\langle z^{m} f_{i}(z), z^{n} f_{j}(z)\right\rangle=\delta_{m n} \delta_{i j},
$$

$m, n=0,1,2, \cdots$, and $i, j=0, \cdots, r$. Let $M$ be the $(r+1) \times(r+1)$ matrix whose $i$ th row vector is

$$
X_{i}=\left(\bar{c}_{1} f_{i}(z), \bar{c}_{2} f_{i}(z), \cdots, \bar{c}_{r} f_{i}(z), \bar{c}_{1} f_{i}(z)\right) .
$$

Since the first and last columns of $M$ coincide, det $M=0$. A standard argument $\left[4\right.$, pp. 23-24] shows that there are cofactors $F_{0}(z), \cdots$, $F_{r}(z)$ of $M$, not all identically zero, such that

$$
F_{0}(z) \cdot X_{0}+\cdots+F_{r}(z) \cdot X_{r}=0 .
$$

The cofactors of $M$ are formal power series with square summable complex coefficients. To prove this, it suffices to show that if $c$ is any $c_{i}$ and if $f(z)$ is any $f_{j}(z)$, then $F(z) \bar{c} f(z)$ is in $\mathcal{K}(z)$ (where $\mathcal{K}$ de- 
notes the complex numbers) whenever $F(z)$ is in $\mathcal{K}(z)$. If $F(z)$ $=\sum \alpha_{n} z^{n}$, then

$$
F(z) \bar{c} f(z)=\sum \alpha_{n} z^{n} \bar{c} f(z)
$$

holds with convergence in the formal sense. By (2),

$$
\left\|\sum_{M}^{N} \alpha_{n} z^{n} \bar{c} f(z)\right\|_{\mathcal{K}_{(z)}}^{2} \leqq|c|^{2}\left\|\sum_{M}^{N} \alpha_{n} z^{n} f(z)\right\|_{\mathcal{C}_{(z)}}^{2}=\sum_{M}^{N}\left|\alpha_{n}\right|^{2} .
$$

It follows that the series in (4) converges in the metric of $\mathcal{K}(z)$, and by the assertion at the beginning of the proof, (4) holds in the metric of $\mathcal{K}(z)$. In particular, $F(z) \bar{c} f(z)$ is in $\mathcal{K}(z)$. Therefore, the cofactors of $M$ have square summable coefficients.

Let $F_{i}(z)=\sum \alpha_{i n} z^{n}$. By (3),

$$
\sum_{i=0}^{r} F_{i}(z) \cdot f_{i}(z)=0
$$

and

$$
\sum\left[\alpha_{0 n} z^{n} f_{0}(z)+\cdots+\alpha_{r n} z^{n} f_{r}(z)\right]=0,
$$

with convergence of this last series in the metric of $\mathfrak{C}(z)$. By (2) we obtain the contradiction that the norm squared of the left-hand side of $(5)$ is

$$
\sum\left(\left|\alpha_{0 n}\right|^{2}+\cdots+\left|\alpha_{r n}\right|^{2}\right)>0 .
$$

This completes the proof that the dimension of $B$ is at most the dimension of $\mathfrak{e}$.

If $\mathscr{T C}$ is the zero ideal, then $\mathfrak{T C}$ is of the form $\mathfrak{T C}(B)$ trivially. If $\mathscr{T C}$ contains a nonzero element, so does $B$. Let $\left(f_{i}(z)\right)_{i \in I}$ be an orthonormal basis for $B$. Since the dimension of $B$ is no more than the dimension of $\mathfrak{C}$, there is an orthonormal set $\left(c_{i}\right)_{i \in I}$ in $\mathfrak{C}$ of the same cardinality. Define a formal power series $B(z)$ with operator coefficients by

$$
B(z) c=\sum \bar{c}_{i} c f_{i}(z) .
$$

It is easy to see that $B(z)$ satisfies (1) with $N(B)$ equal to the orthogonal complement of the $c_{i}(i \in I)$, and that $\mathfrak{T l}(B) \subseteq \mathfrak{T}$.

To complete the proof, we will show that the orthogonal complement $\mathfrak{N}$ of $\mathscr{N}(B)$ in $\mathscr{T}$ is zero. Notice that $B \subseteq \mathscr{T}(B)$ and

$$
\Re=\Re(B) \perp \cap \Re \subseteq B \perp \cap \Re .
$$

Let $f(z)$ be any element of $\Re$. Then $f(z)$ is in $\Re$ and it is orthogonal 
to $B$. By the definition of $B, f(z)=z f_{1}(z)$ for some $f_{1}(z)$ in $\mathfrak{T}$. If $g(z)$ is any element of $\mathfrak{T}(B)$,

$$
\left\langle f_{1}(z), g(z)\right\rangle=\left\langle z f_{1}(z), z g(z)\right\rangle=\langle f(z), g(z)\rangle=0,
$$

since $\mathfrak{T}(B)$ is an ideal. Therefore, $f_{1}(z)$ is in $\Re$. Continuing by induction, we see that for every $r=1,2,3, \cdots$, there is an $f_{r}(z)$ in $\mathfrak{r}$ such that $f(z)=z^{r} f_{r}(z)$. It follows that $f(z)=0$ identically. Therefore $\Re=(0)$ and $\Re=\mathfrak{T}(B)$ as asserted.

Added in proof. The methods of this paper have since been used to obtain the existence of invariant subspaces for transformations $T$ which are bounded by 1 , when $1-T^{*} T$ is completely continuous.

\section{REFERENCES}

1. A. Beurling, On two problems concerning linear transformations in Hilbert space, Acta Math. 81 (1949), 239-255.

2. L. de Branges, Some Hilbert spaces of entire functions, Trans. Amer. Math. Soc. 96 (1960), 259-295. 112.

3. P. R. Halmos, Shifts on Hilbert spaces, J. Reine Angew. Math. 208 (1961), 102-

4. N. Jacobson, Lectures in abstract algebra. II. Linear algebra, The University Series in Higher Mathematics, Van Nostrand, New York, 1953.

5. J. Rovnyak, Ideals of square summable power series, Math. Mag. 33 (1960), 265-270; ibid. 34 (1961), 41-42.

6. Ideals of square summable power series, Proc. Amer. Math. Soc. 13 (1962), 360-365.

7. - Some Hilbert spaces of analytic functions, Thesis, Yale University, New Haven, Conn., 1963.

Purdue University 